\title{
Pollos cuello desnudo alimentados con harina de Morus alba y Cajanus cajan
}

\author{
Naked neck chickens fed with flour Morus alba and Pigeon pea
}

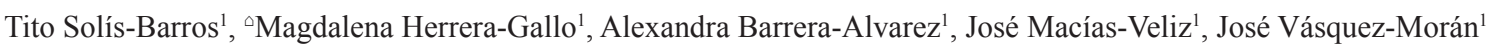

${ }^{1}$ Universidad Técnica Estatal de Quevedo. Facultad de Ciencias Pecuarias. Carrera de Ingeniería Zootecnica. Campus Finca Experimental "La María”. CP. 121250. Km. 7 1/2 vía El Empalme, cantón Mocache. Los Ríos. Ecuador. tsolis@uteq.edu.ec, •sherrera@uteq.edu.ec,jnmacias@uteq.edu.ec; barreraalvarez@yahoo.com; jvasquez@uteq.edu.ec

Rec.: 03.05.2017. Acept.: 31.08.2017.

Publicado el 1 de diciembre de 2017

\section{Resumen}

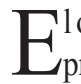
lobjetivo de la investigación fue estudiar el comportamiento productivo de pollos cuello desnudo heterocigotos que se alimentaron con harina de hojas de Morus alba y granos de Cajanus cajan tostados a $120{ }^{\circ} \mathrm{C}$ por $12 \mathrm{~min}$, se utilizaron 144 pollos sexados de la estirpe T451N de 1 a $90 \mathrm{~d}$ de edad. Se distribuyeron según diseño de bloques al azar, en tres tratamientos, 48 pollos por tratamiento repartidos en ocho réplicas. Fueron criados en piso, en instalaciones rústicas. Se evaluaron indicadores productivos como el consumo de alimento, ganancia de peso promedio diario, conversión alimentaria y la eficiencia en el uso de los nutrientes. Los tratamientos fueron: $\mathrm{T} 1=$ Control, $\mathrm{T} 2=5 \%$ C c cajan y $3 \% M$. alba y $\mathrm{T} 3=3 \%$ C. cajan y 5\% M. alba. El control consumió maíz y soya, como dieta base. La mayor ingestión de alimento $(\mathrm{p}<0.05)$ lo presentó el T3 (8884 g). Sin embargo, el T2 registró el mayor peso vivo $(\mathrm{p}<0.05)$ acumulado final/animal $(3250 \mathrm{~g})$ y ganancia promedio diaria de peso vivo $(34 \mathrm{~g})$, valores que resultaron similares al control. En la conversión alimentaria, no se registraron diferencias $(\mathrm{p}>0.05)$ entre tratamientos. Las tres dietas tuvieron similares contenidos nutricionales. Sin embargo, los animales del T2 tuvieron la mayor eficiencia en el uso de la proteína y la energía y de sus dietas. Se concluye que la cría de pollos cuello desnudo heterocigotos, alimentados con dieta con el $5 \%$ de harina de C. cajan y $3 \%$ de harina de hojas de $M$. alba mejoró el comportamiento productivo.

Palabras clave: avicultura, consumo, conversión, pollos cuello desnudos.
$\mathrm{T}^{\mathrm{s}}$ o study the productive behavior of heterozygous naked neck chickens fed with Morus alba leaf meal and Cajanus cajan grains roasted at $120^{\circ} \mathrm{C}$ for $12 \mathrm{~min}$, we used 144 sexed chickens from the $\mathrm{T} 451 \mathrm{~N}$ strain from 1 to $90 \mathrm{~d}$ of age. The chickens were distributed in three treatments, 48 chickens per treatment distributed in eight replicates. According to random block design. The chickens were raised on floor, in rustic facilities. Productive indicators such as food consumption, average daily weight gain, feed conversion and nutrient use efficiency were evaluated. The treatments were: $\mathrm{T} 1=\mathrm{Control}, \mathrm{T} 2=5 \% \mathrm{C}$. cajan and 3\% M. alba and T3=3\% C. cajan and 5\% M. alba. Control consumed maize and soy, as a dietary basis. The $\mathrm{t} 3$ presented the highest food intake $(\mathrm{p}<0.05)(8884 \mathrm{~g})$. However, T2 recorded the highest cumulative live weight $(\mathrm{p}<0.05) /$ animal $(3250$ g) and average daily gain of live weight $(34 \mathrm{~g})$, values that were similar to the control. In feed conversion, there were no significant differences $(p>0.05)$ between treatments. The three diets had similar nutritional contents. However, T2 animals had the greatest efficiency in the energy and protein use of their diets. It was concluded that the breeding of heterozygous naked neck chickens, fed a diet with $5 \%$ of flour of $C$. cajan and 3\% of flour of leaves of $M$. alba, improved the productive behavior.

Key words: poultry farming, consumption, conversion, naked neck chickens. 


\section{Introducción}

$\mathrm{D}$ ado el incremento en el consumo de aves de traspatio y a la escasez de maíz y soja, insumos utilizados en dietas para monogástricos, ha inducido a los investigadores a buscar nuevas alternativas de alimentación (Herrera et al., 2014).

En las zonas tropicales hay una amplia variedad de recursos disponibles que se pueden utilizar en la alimentación de especies monogástricas, entre los que se incluyen fuentes voluminosas con alto contenido fibroso (Savón 2010) y que su utilización en dietas para estos animales, representa una importante contribución económica. No obstante, el nivel de inclusión en las dietas y composición de la fibra afectan el comportamiento productivo y morfofisiológico de ellos (González et al., 2007; Savón, 2010; Herrera et al., 2014). Entre estas alternativas alimenticias se encuentran la harina de hojas de arbustos, como la morera (Morus alba) y el grano tostado de gandul (Cajanus cajan) que podrían reducir el costo de la ceba de pollos (Herrera et al., 2014; Polyana et al., 2014; Herrera et al., 2015), particularmente, los cuello desnudo (guaricos) que poseen alta eficiencia, toleran mejor el calor y las condiciones adversas (Fathi et al., 2008). No obstante, estas plantas poseen factores antinutricionales (FAN) que se pueden reducir con el secado al sol o el tostado (Hirigoyen et al., 2011; Quicazán y Caicedo, 2012), respectivamente. Por todo lo anterior, el objetivo de este trabajo fue evaluar el comportamiento productivo de pollos de cuello desnudo que consumieron dietas con la inclusión de harinas de follaje de M. alba y de granos tostados de C. cajan.

\section{Materiales y Métodos}

$\mathrm{L}$ ocalización. Los Ríos, Ecuador, a $01^{\circ} 06^{\prime}$ latitud sur y $79^{\circ} 29^{\prime}$ latitud oeste, $75 \mathrm{~m}$ sobre el nivel del mar, con temperatura promedio anual de $24.70{ }^{\circ} \mathrm{C}$, humedad del $87 \%$, precipitación promedio anual de $2613 \mathrm{~mm}$, heliofanía de 886 horas y suelo franco arcilloso.

Harinas M. alba (var. Criolla) se plantó con estacas de $40 \mathrm{~cm}$, a una distancia de narigón de $40 \mathrm{~cm}$ y $1 \mathrm{~m}$ de camellón, en un área de $5000 \mathrm{~m}^{2}$ que se dividió en dos lotes $\left(2500 \mathrm{~m}^{2} /\right.$ lote $)$ y se fertilizó con abono orgánico (300 kg/ha/año). El corte inicial de M. alba se realizó luego de un año de establecimiento. El follaje se colectó manualmente, cada 45 días y se secó a la sombra por tres días. C. cajan se sembró con $18 \mathrm{~kg}$ de semilla/ha, al voleo. La recolección de granos se realizó, después de seis meses y se tostaron al carbón, a $120^{\circ} \mathrm{C}$ por $12 \mathrm{~min}$. Ambas materias primas se molieron, para elaborar las harinas.

Tratamientos. En este trabajo se plantearon tres tratamientos experimentales. El control (T1) que consumió maíz y soya, como dieta base. Al tratamiento dos (T2), se le incluyó $5 \%$ C. cajan y $3 \%$ M. alba; y al T3, 3\% C. cajan y $5 \%$ M. alba. Se evaluaron dos fases experimentales: inicial y final.

Análisis químico. Materia seca (MS), proteína cruda (PC), energía metabolizable (EM), fibra cruda (FC), calcio $(\mathrm{Ca})$ y fósforo $(\mathrm{P})$ de los alimentos según, AOAC (2012).

Animales y procedimiento experimental. Los pollos ingresaron con un peso inicial de $48 \mathrm{~g} / \mathrm{ave}$ y un día de edad. Tiempo de experimentación de 1-90 días. Se vacunaron contra Newcastle. Se alojaron en instalaciones rústicas. Se criaron en piso, con cama de viruta de $15 \mathrm{~cm}$. En el Cuadro 1 se presentan las dietas experimentales, en la etapa inicial y final. La etapa inicial de 28 días de duración y la final de 62 días. Los animales se pesaron cada siete días, antes de la ingestión del alimento, para calcular la ganancia media diaria, por fase y acumulada de la crianza. Se ubicaron debajo de calentadoras, por siete días. En cada réplica se instaló un bebedero y comedero manual y un bombillo de 60 watt. Se midió el consumo voluntario (oferta-rechazo), una vez a la semana. Se calcularon la conversión (ingestión/ ganancia de peso) y la eficiencia en el uso de energía y proteína para lo cual utilizaron las siguientes fórmulas:

1. $\mathrm{APC}=\mathrm{Consumo} / \mathrm{dí}{ }^{*} \mathrm{PC} / 100$

2. $\mathrm{EFPC}=\mathrm{APC} / 3 \mathrm{G} / \mathrm{d}$ ía

Donde:

1. $\mathrm{APC}=$ Aporte proteína cruda

2. $\mathrm{EFPC}=$ Eficiencia de proteína cruda $=$ Aporte de $\mathrm{PC} /$ ganancia/día

3. $\mathrm{G}=$ Ganancia promedio diaria de peso vivo (g por animal por día)

Fase inicial: 28 días; Fase final, (crecimiento-ceba): 62 días.

\section{Análisis estadístico.}

Para el análisis de varianza de tres tratamientos con 48 aves/tratamiento, repartidos homogéneamente por peso en ocho réplicas, con seis animales cada una, bajo un diseño de bloques al azar, se utilizó el software SAS v.9.3 (Statistical Analysis System, 2013), para evaluar estadígrafos descriptivos (media y desviación estándar) y la prueba de Tukey, para la comparación de medias. 
Cuadro 1. Dietas de pollos cuello desnudo alimentados con harina de granos tostados de $C$. cajan y hojas de $M$. alba

\begin{tabular}{|c|c|c|c|c|c|c|}
\hline \multirow{3}{*}{$\begin{array}{l}\text { Ingredientes }(\%) \\
\text { Fases de la crianza }\end{array}$} & \multirow{2}{*}{\multicolumn{2}{|c|}{ Control }} & \multicolumn{4}{|c|}{ Harinas } \\
\hline & & & \multicolumn{2}{|c|}{ C. cajan (5\%) y M. alba (3\%) } & \multicolumn{2}{|c|}{ C. cajan (3\%) y M. alba (5\%) } \\
\hline & Inicial & Final & Inicial & Final & Inicial & Final \\
\hline Maíz, harina & 62.12 & 67.62 & 56.09 & 59.58 & 56.23 & 59.58 \\
\hline Soya, harina & 16.97 & 10.00 & 15.28 & 10.00 & 15.00 & 10.00 \\
\hline Gandul, harina & 0.00 & 0.00 & 5.00 & 5.00 & 3.00 & 3.00 \\
\hline Morera, harina & 0.00 & 0.00 & 3.00 & 3.00 & 5.00 & 5.00 \\
\hline Palma, aceite & 1.80 & 3.00 & 1.50 & 3.00 & 1.50 & 3.00 \\
\hline Pescado, harina & 8.50 & 8.50 & 8.50 & 8.50 & 8.50 & 8.50 \\
\hline Alfalfa, harina & 5.00 & 5.00 & 5.00 & 5.00 & 5.00 & 5.00 \\
\hline Arroz, polvo & 3.00 & 3.12 & 3.00 & 3.12 & 3.12 & 3.12 \\
\hline Fosfato di-cálcico & 0.62 & 0.70 & 0.70 & 0.70 & 0.70 & 0.70 \\
\hline Pre-mezcla ${ }^{1}$ & 0.25 & 0.25 & 0.25 & 0.25 & 0.25 & 0.25 \\
\hline Carbonato de calcio & 1.10 & 0.95 & 0.95 & 0.95 & 0.95 & 0.95 \\
\hline Cloruro de sodio & 0.20 & 0.20 & 0.20 & 0.20 & 0.20 & 0.20 \\
\hline Ácido propiónico & 0.05 & 0.05 & 0.05 & 0.05 & 0.05 & 0.05 \\
\hline Antioxidante & 0.05 & 0.05 & 0.05 & 0.05 & 0.05 & 0.05 \\
\hline Lisina & 0.18 & 0.45 & 0.28 & 0.45 & 0.25 & 0.45 \\
\hline Metionina & 0.11 & 0.06 & 0.10 & 0.10 & 0.15 & 0.10 \\
\hline Formol & 0.05 & 0.05 & 0.05 & 0.05 & 0.05 & 0.05 \\
\hline Total & 100.00 & 100.00 & 100.00 & 100.00 & 100.00 & 100.00 \\
\hline Indicadores & & Com & osición quín & le las die & & \\
\hline Materia seca (\%) & 85.00 & 86.00 & 87.00 & 85.00 & 86.00 & 88.00 \\
\hline Proteína cruda (g) & 20.00 & 18.40 & 20.20 & 18.50 & 20.30 & 18.50 \\
\hline $\mathrm{EM}(\mathrm{MJ} / \mathrm{kg})$ & 12.70 & 13.00 & 12.50 & 12.90 & 12.40 & 12.60 \\
\hline Fibra cruda (\%) & 3.00 & 3.30 & 3.20 & 3.90 & 3.50 & 4.30 \\
\hline Calcio (\%) & 0.90 & 0.90 & 0.90 & 0.90 & 1.00 & 1.10 \\
\hline Fósforo (\%) & 0.40 & 0.40 & 0.40 & 0.40 & 0.40 & 0.50 \\
\hline
\end{tabular}

Un kg de alimento contiene: suplemento vitamínico: Vitamina A (10000 UI), Vitamina D3 (2000 UI), Vitamina E (10 mg), Vitamina K 3 (2 mg), Tiamina (1 mg)-B1, Riboflavina (5 mg), Piridoxina (2 mg)- B6, Vitamina B12 (15 mg), Ácido nicotínico (125 mg), Pantotenato de calcio $(10 \mathrm{mg})$, Ácido fólico $(0.25 \mathrm{mg})$, y Biotina $(0.02 \mathrm{mg})$. Suplemento mineral: Selenio mineral: Selenio (0.1 mg), Hierro (40 mg), Cobre (12 mg), Zinc (120 mg), Mg (100 mg), Yodo (2.5 mg) y Cobalto (0.75 mg). (INIAP), Quito, Ecuador.

Fase Inicial: (28 días); Fase final: crecimiento-ceba (62 días).

\section{Resultados y Discusión}

$\mathrm{L}$ a inclusión de C. cajan (3\%) y M. alba (5\%) (T3) registró la mayor ingestión de alimento $(\mathrm{P}<0.05)$ en pollos camperos (8884 g). Estos resultados fueron superiores a los presentados por Olmo et al. (2012), al utilizar $10 \%$ M. alba (3859 g) y similares (7420 g) a los reportados por Herrera (2014) con el 6\% M. alba.

Por otra parte, Polyana et al. (2014) suministraron niveles del 5 al $10 \%$ de $C$. cajan a pollos de engorde, obteniendo valores de 2626 - $2640 \mathrm{~g}$ con una conversión de 2.43 con la inclusión del 5\% de C. cajan; resultados similares obtenidos por Zambrano y Zambrano (2014) quienes utilizaron el mismo nivel de C. cajan. Esto es, la fibra aumenta la ingestión, motilidad gastrointestinal pero reduce la digestibilidad, y pudiera deberse a la presencia de FAN que pueden interferir directamente en la disponibilidad de aminoácidos esenciales como lisina y metionina y por ende afectar directamente a la ganancia de peso (Navarro et al., 2014).

El mayor peso vivo, ganancia promedio diaria de 
peso vivo $(\mathrm{p}<0.05)$ y eficiencia de la proteína y de la energía, fueron obtenidos por los pollos que consumieron C. cajan (5\%) y M. alba (3\%) (T2), valores que fueron similares al control (T1), queda demostrado que fueron más eficientes en el uso de la energía y de la proteína de sus dietas aunque, no se observaran diferencias significativas en las dos fases (crecimiento y final) (Cuadro 2). Estos resultados podrían deberse por la dieta que tuvo menor presencia de factores antinutricionales (FAN) lo que coincide con lo manifestado por Sánchez y Truque (2015) quienes publican que la presencia de FAN puede interferir directamente en la disponibilidad de aminoácidos azufrados como metionina, triptófano, lisina, lo que pudiera impedir que el animal demuestre su verdadero potencial productivo.

Según Albert y Rodríguez (2014), la M. alba tiene bajo contenido de fibra y factores antinutricionales reductores del consumo, pero, un alto valor biológico que pudiera potenciar la palatabilidad representada en su mayor ganancia diaria de peso vivo. Por su parte, De Luna (2006) detectó que el tostado de granos aumentó la disponibilidad de nutrientes, al eliminar los FAN termolábiles y la mayoría de los FAN de C. cajan son termolábiles (Sánchez y Truque 2015).

Mukhtar et al. (2013) obtuvieron menor peso vivo final $(2129.38 \mathrm{~g})$ y ganancia de peso acumulada por animal (1967.50 g), con $20 \%$ C. cajan. Al hafiz et al. (2013) lograron $2085 \mathrm{~g}$ con $12 \%$ C. cajan decorticado. Chambilla (2012) consiguió $2536.90 \mathrm{~g}$ de ganancia acumulada, con $15 \%$ C. cajan. Es probable que las diferencias se debieran a la forma y porcentaje del uso de los granos en su dieta.

El peso vivo final fue similar a los valores obtenidos por Herrera et al. (2014) con $3330 \mathrm{~g}$, con 3\% M. alba e Itzá et al. (2010) y Casamachín et al. (2007) con 4 y 5\%. Trompiz et al. (2011) y Labrador y Andara (2012) utilizaron el $20 \%$ C. cajan y los indicadores productivos se afectaron, pues, queda demostrado que en aves se deben utilizar niveles bajos de fibra dado la conformación anatómica digestiva de las aves (Polyana et al, 2014).

Cuadro 2. Comportamiento productivo y eficiencia en el uso de energía y proteína

\begin{tabular}{|c|c|c|c|c|c|}
\hline $\begin{array}{l}\text { Tratamientos } \\
\text { Indicadores }\end{array}$ & $\begin{array}{c}1 \\
\text { Control }\end{array}$ & $\begin{array}{c}2 \\
\text { C. cajan }(5 \%)+ \\
\text { M. alba }(3 \%)\end{array}$ & $\begin{array}{c}3 \\
\text { C. cajan }(3 \%)+ \\
\text { M. alba }(5 \%)\end{array}$ & $\begin{array}{l}\mathbf{E E} \\
( \pm)\end{array}$ & Significación \\
\hline \multicolumn{6}{|c|}{ Ingestión de alimento (g/animal) } \\
\hline Fase Inicial & 1099.16 & 1107.12 & 1136.14 & 42.13 & 0.152 \\
\hline Fase final & $7478.24 a$ & $7509.28 \mathrm{a}$ & $7748.54 b$ & 92.31 & 0.057 \\
\hline Total & $8577.40 \mathrm{a}$ & $8616.40 \mathrm{a}$ & $8884.68 b$ & 69.56 & 0.000 \\
\hline \multicolumn{6}{|c|}{ Peso vivo acumulado (g) } \\
\hline Fase Inicial & 830.45 & 835.12 & 840.2 & 20.32 & 0.147 \\
\hline Fase final & $3200.42 \mathrm{a}$ & $3250.25 \mathrm{a}$ & $2845.22 b$ & 32.10 & 0.000 \\
\hline \multicolumn{6}{|c|}{ Ganancias promedio diaria de peso vivo (g por animal por día) } \\
\hline Fase Inicial & 29.66 & 29.83 & 30.01 & - & - \\
\hline Fase final & 37.90 & 38.95 & 32.34 & - & - \\
\hline Total & $33.78 \mathrm{a}$ & $34.39 \mathrm{a}$ & $31.17 \mathrm{~b}$ & 0.34 & 0.000 \\
\hline \multicolumn{6}{|c|}{ Conversión de alimentos } \\
\hline Fase Inicial & 1.39 & 1.32 & 1.5 & 0.02 & 0.123 \\
\hline Fase final & 2.34 & 2.31 & 3.86 & 0.04 & 0.132 \\
\hline \multicolumn{6}{|c|}{ Eficiencia en el uso de la proteína (PC del aporte/ganancia media diaria) (g/g) } \\
\hline Fase Inicial & 2.69 & 2.67 & 2.74 & - & - \\
\hline Fase final & 5.91 & 5.74 & 7.15 & - & - \\
\hline \multicolumn{6}{|c|}{ Eficiencia en el uso de la energía (EM del aporte/ganancia media diaria) (MJ/g) } \\
\hline Fase Inicial & 3.97 & 3.96 & 4.01 & - & - \\
\hline Fase final & 9.90 & 9.59 & 11.66 & - & - \\
\hline
\end{tabular}

Medias con una letra común, no son significativamente diferentes (Tukey, p>0.05) 
Los resultados obtenidos pudieran deberse a la combinación de una leguminosa y una arbustiva incorporada en dietas para pollos rústicos mejorados cuello desnudo heterocigotos. Navarro et al. (2014) manifiesta que el $C$. cajan es deficiente en los aminoácidos azufrados (lisina, metionina y triptófano), que lo compensa muy bien la M. alba, cuyas hojas fueron cortadas a los 45 días cuando su contenido proteico es muy alto y bajo en metabolitos secundarios (Olmo et al., 2012) asociado con pollos que toleran mejor el calor por la reducción del plumaje (Galal et al., 2007) y utilizan mejor la energía y la proteína, porque requieren de 2-3\% menos proteína que los de plumaje completo (Fathi et al., 2008) logrando optimizar la utilización de la energía y la proteína de las dietas, para transformarlas en ganancia de peso vivo.

\section{Conclusiones}

$E^{s}$ s posible utilizar $5 \%$ de granos tostados de C. cajan, a $120^{\circ} \mathrm{C}$ por $12 \mathrm{~min}$, más $3 \%$ de harina de hojas de $M$. alba, para sustituir maíz y soya en la dieta de pollos cuello desnudo y obtener un favorable consumo (8614.40 g); peso vivo final (3250.25 g), conversión alimentaria (2.31) y eficiencia en el uso de nutrientes (energía y proteína).

\section{Bibliografía}

Al hafiz, AH., Elshiek, OY., and Gibril, S. (2013). Effect of feeding graded levels of decorticated Pigeon pea (Cajanus cajan) seeds on broiler chicks performance. J. of Applied and Industrial Sc. 1(4): 7-10.

Albert, A., Rodríguez, Y. (2014). Estudio de los factores antinutricionales de las especies Morus alba Lin (morera), Trichanthera gigantea (h y b), nacedero; y Erythrina poeppigiana (Walp. O. F), piñón para la alimentación animal. Rev. Acad. de Inv. TLATEMOANI. 17: 1-15.

AOAC. (2012). Official Methods of Analysis (19 th) Ass. Off. Anal. Chem. Arligton, VA. Washington, D.C. http://www.worldcat.org/title/officialmethod s-of-analysis-of-aoac-international/oclc/8 55542981 ?referer $=$ di\&ht $=$ edition.

Casamachín, M., Ortiz, D., y López, J. (2007). Evaluación de tres niveles de inclusión de morera (Morus alba) en alimento para pollos de engorde. Rev. Biot. Sector Agrop. Agroind. 5(2): 64-71.

Chambilla, CE. (2012). Efecto de tres niveles de harina de semilla de gandul (Cajanus cajan L. Millsp), en el crecimiento de pollos parrilleros de la línea Ross 308 en el cantón Santa Fe de la provincia Caranavi. Tesis de Titulación. Universidad Mayor de San Andrés, La Paz, Bolivia, 107p.

De Luna, JA. (2006). Valor nutritivo de la proteína de soya. Rev. Invest. y Cienc. de la Universidad Autónoma de Aguascalientes, México. 36: 29-34.

Fathi, M., Elattar, A., Ali, U., and Nazmi, A. (2008). Effect of the naked neck gene on carcase composition and immunocompetence in chicken. Rev. British Poult. Sc. 49(2): 103-110.

Galal, A., Ahmed, A., Ali, UM., Younis, HH. (2007). Influence of Naked Neck Geneon Laying Performance and Some Hematological Parameters of Dwarfing Hens. Int. J. Poult. Sc. 6 (11): 807813

Herrera, GSM., Díaz, CA., Muñoz, RG., Espinoza, GI., Romero, RJ., and Solís, BT. (2015). Productive performance of Rhode Island Red hens fed with foliage shrubs. Global Advanced Res. J. of Agric. Sc. 4(10): 673-676.

Herrera, SM, Savón, L., Lon-Wo, E., Gutiérrez, O., y Herrera, M. (2014). Inclusión de harina de hojas de Morus alba: su efecto en la retención aparente de nutrientes, comportamiento productivo $\mathrm{y}$ calidad de la canal de pollos cuello desnudo. Rev. Cub. Cienc. Agríc. 48(3): 259-264.

Herrera, GSM. (2014). Caracterización y manejo de un sistema de alimentación alternativo en pastoreo, para pollos cuello desnudo heterocigotos. Tesis de Doctor en Ciencias Veterinarias. Instituto de Ciencia Animal, Universidad Agraria de La Habana, Cuba, 165p.

Hirigoyen, C., Bratschi, S., Furtado, G., Arias, A., González, R., y Bauza, R. (2011). Caracterización química del grano de soja sometido a diferentes tratamientos de desactivación. Efecto del cocimiento en agua. Resúmenes del simposio, UDELAR, Uruguay, $205 \mathrm{p}$.

Itzá, M., Lara, P., Magaña, MA., y Sanginés, J. (2010). Evaluación de la harina de hoja de morera (Morus $a l b a)$ en la alimentación de pollos de engorda. Zootecnia Trop. 28(4): 477-488.

Labrador, J., y Andara, J. (2012). Incorporación del quinchoncho (Cajanus cajan) en raciones alimenticias para pollos de engorde. Revista Científica, FCV-LUZ, XXII (Suplemento 1): 2728.

Mukhtar, A., Mukhtar, AE., and Amal, O. (2013). Response of broiler chicks fed on diets containing graded levels of pigeon pea (Cajanus cajan) seeds supplemented with essential caraway oil. J. of Current Res. in Sc. 1(2): 109-112. 
Navarro, CL., Restrepo, MD., and Pérez, J. (2014). El guandul (Cajanus cajan) una alternativa en la industria de los alimentos. Biot. Sect. Agrop. Agroind. 12(2): 197-206.

Olmo, C., Martínez, Y., León, E., Leyva, L., Nuñez, M., Rodríguez, R., Labrada, A., Isert, M., Betancur, C., Merlos, C., and Liu, G. (2012). Effect of Mulberry Foliage (Morus alba) Meal on Growth Performance and Edible Portions in Hybrid Chickens. Int. J. An. and Vet. Adv. 4(4): 263.

Polyana, AD., Maciel, MP., Rocha, LFB., Albuquerque, LC., Martins, LFS., Batista, DS., e Santana, VH. de M. (2014). Feijão guandu cru na alimentação de frangos caipiras criados em sistema semi-intensivo. Revista Pesq. Agropec. Bras. 49(9): 737-744.

Quicazán, M., y Caicedo, L. (2012). Inactivación del inhibidor de tripsina, durante el tratamiento térmico de bebidas de soya. Artículo corto. Rev. Vitae 19 (Sup.1): 337-339.

Rodríguez, R., Martínez, M., Valdivié, M., y Cisneros, M. (2006). Morfometría del tracto gastrointestinal y sus órganos accesorios en gallinas ponedoras alimentadas con piensos que contienen harina de caña proteica. Rev. Cub. Cienc. Agríc. 40(3): 361365.
Sánchez, J. y Truque, Y. (2015). Evaluación de la inclusión de harina de guandul (Cajanus cajan) en la alimentación de pollos de engorde [Tesis pregrado Administrador de empresas agropecuarias]. Popayán (Colombia): Fundación Universitaria de Popayán, Facultad Ciencias Económicas, Contables y Administrativas, $90 \mathrm{p}$.

SAS. (2013). Statistics. v. w. 9.3. De SAS Institute. INC. Cary. N. C. USA.

Trómpiz, J., Rincón, H., Fernández, N., González, G., Higuera, A., y Colmenares, C. (2011). Parámetros productivos en pollos de engorde alimentados con grano de quinchoncho durante fase de crecimiento. Rev. Fac. Agr. (LUZ). 28 (Supl. 1): 565-575.

Zambrano, FRF., y Zambrano, MJR. (2014). Inclusión de harina de frejol de palo (Cajanus cajan L, Mill sp.) en el alimento de pollos de engorde y su efecto en parámetros productivos. Tesis de Médico Veterinario. Escuela Superior Politécnica Agropecuaria de Manabí. 76 p. 